\title{
Facile $\boldsymbol{O}$-Arylation of Phenols and Carboxylic Acids
}

\author{
Zhijian Liu and Richard C. Larock * \\ Department of Chemistry, Iowa State University, Ames, Iowa 50011
}

\section{Supporting Information}

General. The ${ }^{1} \mathrm{H}$ and ${ }^{13} \mathrm{C}$ NMR spectra were recorded at 300 and $75.5 \mathrm{MHz}$ or 400 and $100 \mathrm{MHz}$ respectively. All melting points are uncorrected. High resolution mass spectra were recorded on a Kratos MS50TC double focusing magnetic sector mass spectrometer using EI at $70 \mathrm{eV}$. All reagents were used directly as obtained commercially unless otherwise noted. All yields reported in the publication represent an average of at least two independent runs. CsF, acetonitrile and silyaryl triflate 1a were purchased from Sigma-Aldrich Co. The methoxy-substituted silylaryl triflate $\mathbf{1 b}^{1}$ and 4,5-dimethyl-substituted silylaryl triflate $1 \mathbf{c}^{2}$ was prepared according to a previous literature procedure.

\section{General procedure for the $\boldsymbol{O}$-arylation of phenols and carboxylic acids.}

Procedure A: To a solution of $\mathrm{MeCN}(4 \mathrm{~mL})$, the phenol $(0.25 \mathrm{mmol})$ and the silylaryl triflate $(0.375 \mathrm{mmol})$ was added $\mathrm{CsF}(0.75 \mathrm{mmol})$. The reaction mixture was allowed to stir at room temperature for $1 \mathrm{~d}$ and the resulting solution was washed with brine $(20 \mathrm{~mL})$ and extracted with diethyl ether $(20 \mathrm{~mL})$. The combined ether fractions were dried over $\mathrm{Na}_{2} \mathrm{SO}_{4}$ and concentrated under reduced pressure. The residue was purified by flash chromatography on silica gel to afford the desired product.

Procedure B: To a solution of MeCN (4 mL), the carboxylic acid (0.25 mmol) and the silylaryl triflate $(0.5 \mathrm{mmol})$ was added $\mathrm{CsF}(1.0 \mathrm{mmol})$. The reaction mixture was allowed to stir at room temperature for $1 \mathrm{~d}$ and the resulting solution was washed with brine $(20 \mathrm{~mL})$ and extracted with diethyl ether $(20 \mathrm{~mL})$. The combined ether fractions were dried over $\mathrm{Na}_{2} \mathrm{SO}_{4}$ and concentrated under reduced pressure. The residue was purified by flash chromatography on silica gel to afford the desired product. 
Diphenyl ether (entry 1, Table 1). Using procedure A, phenol (0.25 mmol, $24 \mathrm{mg})$, silylaryl triflate 1a $(0.375 \mathrm{mmol}, 112 \mathrm{mg})$ and $\mathrm{CsF}(0.75 \mathrm{mmol}, 114 \mathrm{mg})$ afforded the indicated compound (39 mg) in a $92 \%$ yield as a colorless oil. The ${ }^{1} \mathrm{H}$ and ${ }^{13} \mathrm{C}$ NMR spectra match the literature data: ${ }^{3}{ }^{1} \mathrm{H} \mathrm{NMR}\left(300 \mathrm{MHz}, \mathrm{CDCl}_{3}\right) \delta 7.37-7.32(\mathrm{~m}, 4 \mathrm{H}), 7.14-$ 7.08 (m, 2H), 7.05-7.01 (m, 4H); ${ }^{13} \mathrm{C}$ NMR (75 MHz, $\left.\mathrm{CDCl}_{3}\right) \delta 157.4$ (2C), 129.9 (2C), $123.4(2 \mathrm{C}), 119.1(2 \mathrm{C})$.

4-Methoxyphenyl phenyl ether (entry 2, Table 1). Using procedure A, 4methoxyphenol $(0.25 \mathrm{mmol}, 31 \mathrm{mg})$, silylaryl triflate $1 \mathbf{a}(0.375 \mathrm{mmol}, 112 \mathrm{mg})$ and $\mathrm{CsF}$ $(0.75 \mathrm{mmol}, 114 \mathrm{mg})$ afforded the indicated compound $(49 \mathrm{mg})$ in a $98 \%$ yield as a colorless oil. The ${ }^{1} \mathrm{H}$ and ${ }^{13} \mathrm{C}$ NMR spectra match the literature data: ${ }^{4}{ }^{1} \mathrm{H}$ NMR (300 $\left.\mathrm{MHz} \mathrm{CDCl}_{3}\right) \delta$ 7.34-7.29 (m, 2H), 7.08-6.88 (m, 7H), $3.82(\mathrm{~s}, 3 \mathrm{H}) ;{ }^{13} \mathrm{C}$ NMR $(75 \mathrm{MHz}$, $\left.\mathrm{CDCl}_{3}\right) \delta 158.7$ (1C), 156.1 (1C), 150.3 (1C), 129.8 (2C), 122.6 (1C), 121.1 (2C), 117.8 (2C), 115.1 (2C), 55.8 (1C); IR $\left(\mathrm{CDCl}_{3}\right)$ 3040, 2952, 1589, $1225 \mathrm{~cm}^{-1}$.

3,4-Dimethyphenyl 4-methoxyphenyl ether (entry 3, Table 1). Using procedure A, 4methoxyphenol (0.25 mmol, $31 \mathrm{mg})$, silylaryl triflate 1c $(0.375 \mathrm{mmol}, 122 \mathrm{mg})$ and CsF (0.75 mmol, $114 \mathrm{mg})$ afforded the indicated compound (54 $\mathrm{mg})$ in a $96 \%$ yield as a colorless oil. The ${ }^{1} \mathrm{H}$ and ${ }^{13} \mathrm{C}$ NMR spectra match the literature data: ${ }^{5}{ }^{1} \mathrm{H}$ NMR (300 $\left.\mathrm{MHz}, \mathrm{CDCl}_{3}\right) \delta 7.04(\mathrm{~d}, J=8.1 \mathrm{~Hz}, 1 \mathrm{H}), 6.95-6.81(\mathrm{~m}, 4 \mathrm{H}), 6.77-6.65$ (m, 2H), 3.76 (s, 3H), 2.20 (s, 6H); ${ }^{13} \mathrm{C}$ NMR (75 MHz, $\left.\mathrm{CDCl}_{3}\right) \delta 156.1$ (1C), 155.4 (1C), 150.6 (1C), 137.9 (1C), 130.5 (1C), 130.1 (1C), 120.1 (2C), 119.1 (1C), 115.0 (1C), 114.7 (2C), 55.3 (1C), 19.7 (1C), 18.7 (1C); IR ( $\left.\mathrm{CDCl}_{3}\right)$ 2931, 2837, 1506, 1497, 1284, $1178 \mathrm{~cm}^{-1}$.

\section{4-Phenoxybenzaldehyde (entry 4, Table 1). Using procedure A, 4-}

hydroxybenzaldehyde $(0.25 \mathrm{mmol}, 31 \mathrm{mg})$, silylaryl triflate $\mathbf{1 a}(0.375 \mathrm{mmol}, 112 \mathrm{mg})$ and $\mathrm{CsF}(0.75 \mathrm{mmol}, 114 \mathrm{mg})$ afforded the indicated compound $(45 \mathrm{mg})$ in a $91 \%$ yield as a colorless oil. The ${ }^{1} \mathrm{H}$ and ${ }^{13} \mathrm{C}$ NMR spectra match the literature data: ${ }^{1} \mathrm{H}$ NMR (300 $\left.\mathrm{MHz}, \mathrm{CDCl}_{3}\right) \delta 9.92(\mathrm{~s}, 1 \mathrm{H})$, 7.86-7.82 (m, 2H), 7.44-7.38 (m, 2H), 7.25-7.20 (m, 1H), 7.10-7.04 (m, 4H); ${ }^{13} \mathrm{C}$ NMR (75 MHz, $\left.\mathrm{CDCl}_{3}\right) \delta 190.9$ (1C), 163.4 (1C), 155.3 (1C), 132.1 (2C), 131.5 (1C), 130.3 (2C), 125.1 (2C), 120.6 (2C), 117.8 (2C); IR $\left(\mathrm{CDCl}_{3}\right)$ 3061, 2827, 1694, 1584, $1488 \mathrm{~cm}^{-1}$; HRMS m/z 198.0683 (calcd $\mathrm{C}_{13} \mathrm{H}_{10} \mathrm{O}_{2}, 198.0680$ ). 4-Nitrophenyl phenyl ether (entry 5, Table 1). Using procedure A, 4-nitrophenol (0.25 mmol, $35 \mathrm{mg})$, silylaryl triflate $1 \mathbf{a}(0.375 \mathrm{mmol}, 112 \mathrm{mg})$ and $\mathrm{CsF}(0.75 \mathrm{mmol}, 114 \mathrm{mg})$ 
afforded the indicated compound $(52 \mathrm{mg})$ in a $96 \%$ yield as a yellow solid: $\mathrm{mp} 58-59{ }^{\circ} \mathrm{C}$ (lit. $\left.{ }^{7} \mathrm{mp} 58-59{ }^{\circ} \mathrm{C}\right)$. The ${ }^{1} \mathrm{H}$ NMR spectrum matchs the literature data: ${ }^{8}{ }^{1} \mathrm{H}$ NMR (300 $\mathrm{MHz}_{\mathrm{CDCl}} \mathrm{CD} \delta$ 8.21-8.18 (m, 2H), 7.46-7.41 (m, 2H), 7.28-7.25 (m, 1H), 7.11-7.07 (m, 2H), 7.02-6.99 (m, 2H); ${ }^{13} \mathrm{C}$ NMR (75 MHz, CDCl 3 ) $\delta 163.6$ (1C), 154.9 (1C), 142.8 (1C), 130.5 (2C), 126.2 (2C), 125.6 (1C), 120.7 (2C), 117.3 (2C); IR $\left(\mathrm{CDCl}_{3}\right)$ 3110, 3076, 1582, 1486, $1247 \mathrm{~cm}^{-1}$; HRMS m/z 215.2042 (calcd $\mathrm{C}_{12} \mathrm{H}_{9} \mathrm{NO}_{3}, 215.2047$ ).

3-Methoxyphenyl 4-nitrophenyl ether (entry 6, Table 1). Using procedure A, 4nitrophenol $(0.25 \mathrm{mmol}, 35 \mathrm{mg})$, silylaryl triflate $\mathbf{1 b}(0.375 \mathrm{mmol}, 123 \mathrm{mg})$ and CsF (0.75 mmol, $114 \mathrm{mg}$ ) afforded the indicated compound (59 $\mathrm{mg})$ in a $96 \%$ yield as a white solid: $\mathrm{mp} 86-87{ }^{\circ} \mathrm{C}$ (lit. ${ }^{9} \mathrm{mp} 85^{\circ} \mathrm{C}$ ); ${ }^{1} \mathrm{H}$ NMR (300 MHz, $\left.\mathrm{CDCl}_{3}\right) \delta 8.21-8.17$ (m, 2H), $7.32(\mathrm{t}, J=8.1 \mathrm{~Hz}, 1 \mathrm{H}), 7.05-7.00(\mathrm{~m}, 2 \mathrm{H}), 6.82-6.78(\mathrm{~m}, 1 \mathrm{H}), 6.68-6.65(\mathrm{~m}, 2 \mathrm{H})$, $3.81(\mathrm{~s}, 3 \mathrm{H}) ;{ }^{13} \mathrm{C} \mathrm{NMR}\left(75 \mathrm{MHz}, \mathrm{CDCl}_{3}\right) \delta 163.4$ (1C), 161.5 (1C), 156.0 (1C), 142.9 (1C), 130.9 (1C), 126.1 (2C), 117.4 (2C), 112.7 (1C), 111.2 (1C), 106.7 (1C), 55.7 (1C); IR $\left(\mathrm{CDCl}_{3}\right)$ 3077, 2941, 1592, 1486, $1345 \mathrm{~cm}^{-1}$; HRMS m/z 245.0691 (calcd $\mathrm{C}_{13} \mathrm{H}_{11} \mathrm{NO}_{4}$, 245.0688).

4-Iodophenyl phenyl ether (entry 7, Table 1). Using procedure A, 4-iodophenol (0.25 mmol, $55 \mathrm{mg})$, silylaryl triflate 1a $(0.375 \mathrm{mmol}, 112 \mathrm{mg})$ and CsF (0.75 mmol, $114 \mathrm{mg})$ afforded the indicated compound $(70 \mathrm{mg})$ in a $95 \%$ yield as a white solid: $\mathrm{mp} 47-48{ }^{\circ} \mathrm{C}$ (lit. $\left.{ }^{10} \mathrm{mp} 48{ }^{\circ} \mathrm{C}\right) ;{ }^{1} \mathrm{H}$ NMR $\left(300 \mathrm{MHz}, \mathrm{CDCl}_{3}\right) \delta$ 7.62-7.58 (m, 2H), 7.37-7.31 (m, 2H), 7.14-7.09 (m, 1H), 7.01-6.98 (m, 2H), 6.78-6.74 (m, 2H); ${ }^{13} \mathrm{C}$ NMR (75 MHz, CDCl 3$) \delta$ 157.7 (1C), 156.7 (1C), 138.8 (2C), 130.1 (2C), 124.0 (1C), 121.1 (2C), 119.4 (2C), 86.1 (1C); IR $\left(\mathrm{CDCl}_{3}\right)$ 3040, 1571, 1477, $1239 \mathrm{~cm}^{-1}$; HRMS m/z 295.9705 (calcd $\mathrm{C}_{12} \mathrm{H}_{9} \mathrm{IO}$, 295.9698).

4-Iodophenyl 3-methoxyphenyl ether (entry 8, Table 1). Using procedure A, 4iodophenol (0.25 mmol, $55 \mathrm{mg})$, silylaryl triflate $\mathbf{1 b}(0.375 \mathrm{mmol}, 123 \mathrm{mg})$ and CsF (0.75 $\mathrm{mmol}, 114 \mathrm{mg}$ ) afforded the indicated compound $(76 \mathrm{mg})$ in a $94 \%$ yield as a colorless oil: ${ }^{1} \mathrm{H}$ NMR (300 MHz, $\left.\mathrm{CDCl}_{3}\right) \delta$ 7.62-7.59 (m, 2H), 7.25-7.20 (m, 1H), 6.79-6.76 (m, 2H), 6.69-6.65 (m, 1H), 6.59-6.55 (m, 2H), 3.77 (s, 3H); $\left.{ }^{13} \mathrm{C} \mathrm{NMR} \mathrm{(75} \mathrm{MHz,} \mathrm{CDCl} 3\right) \delta$ 161.2 (1C), 158.0 (1C), 157.4 (1C), 138.8 (2C), 130.5 (1C), 121.2 (2C), 111.4 (1C), 109.6 (1C), 105.3 (1C), 86.3 (1C), 55.6 (1C); IR ( $\left.\mathrm{CDCl}_{3}\right)$ 3062, 2956, 2833, 1580, 1479, $1272 \mathrm{~cm}^{-1}$; HRMS m/z 325.9792 (calcd $\mathrm{C}_{13} \mathrm{H}_{11} \mathrm{IO}_{2}, 325.9803$ ). 
o-Diphenoxybenzene (entry 9, Table 1). Using procedure A, 1,2-benzenediol (0.25 mmol, $28 \mathrm{mg})$, silylaryl triflate $1 \mathbf{a}(0.75 \mathrm{mmol}, 223 \mathrm{mg})$ and CsF (1.5 mmol, $228 \mathrm{mg})$ afforded the indicated compound $(47 \mathrm{mg})$ in a $72 \%$ yield as a white solid: $\mathrm{mp} 88-90{ }^{\circ} \mathrm{C}$; ${ }^{1} \mathrm{H}$ NMR (300 MHz, $\left.\mathrm{CDCl}_{3}\right) \delta$ 7.29-7.23 (m, 4H), 7.13-7.00 (m, 6H), 6.92-6.88 (m, 4H); ${ }^{13} \mathrm{C}$ NMR (75 MHz, CDCl $) \delta 157.7$ (2C), 148.0 (2C), 129.7 (4C), 124.9 (2C), 123.0 (2C), 121.8 (2C), 117.8 (4C); IR ( $\left.\mathrm{CDCl}_{3}\right)$ 3063, 3038, 1583, 1487, $1262 \mathrm{~cm}^{-1} ; \mathrm{HRMS} \mathrm{m} / \mathrm{z}$ 262.0999 (calcd $\mathrm{C}_{18} \mathrm{H}_{14} \mathrm{O}_{2}, 262.0994$ ).

Phenyl 2,4,6-trimethylphenyl ether (entry 10, Table 1). Using procedure A, 2,4,6trimethylphenol $(0.25 \mathrm{mmol}, 34 \mathrm{mg})$, silylaryl triflate $1 \mathrm{a}(0.375 \mathrm{mmol}, 112 \mathrm{mg})$ and $\mathrm{CsF}$ $(0.75 \mathrm{mmol}, 114 \mathrm{mg})$ afforded the indicated compound $(36 \mathrm{mg})$ in a $68 \%$ yield as a colorless oil: ${ }^{1} \mathrm{H}$ NMR (300 MHz, $\left.\mathrm{CDCl}_{3}\right) \delta$ 7.26-7.21 (m, 2H), 6.97-6.89 (m, 3H), 6.77$6.74(\mathrm{~m}, 2 \mathrm{H}), 2.30(\mathrm{~s}, 3 \mathrm{H}), 2.08(\mathrm{~s}, 6 \mathrm{H}) ;{ }^{13} \mathrm{C} \mathrm{NMR}\left(75 \mathrm{MHz}, \mathrm{CDCl}_{3}\right) \delta 158.2(1 \mathrm{C}), 149.0$ (1C), 134.6 (1C), 131.3 (2C), 129.8 (2C), 129.7 (2C), 121.3 (1C), 114.8 (2C), 21.0 (1C), 16.4 (2C); IR $\left(\mathrm{CDCl}_{3}\right)$ 3025, 2919, 1594, 1482, $1223 \mathrm{~cm}^{-1}$; HRMS m/z 212.1204 (calcd $\left.\mathrm{C}_{15} \mathrm{H}_{16} \mathrm{O}, 212.1201\right)$.

Phenyl benzoate (entry 11, Table 1). Using procedure B, benzoic acid ( $0.25 \mathrm{mmol}, 31$ $\mathrm{mg})$, silylaryl triflate $1 \mathrm{a}(0.5 \mathrm{mmol}, 149 \mathrm{mg})$ and $\mathrm{CsF}(1.0 \mathrm{mmol}, 152 \mathrm{mg})$ afforded the indicated compound $(40 \mathrm{mg})$ in an $81 \%$ yield as a white solid: $\mathrm{mp} 69-70{ }^{\circ} \mathrm{C}$ (lit. ${ }^{11} \mathrm{mp} 68$ $\left.70{ }^{\circ} \mathrm{C}\right)$. The ${ }^{1} \mathrm{H}$ and ${ }^{13} \mathrm{C}$ NMR spectra match the literature data: ${ }^{12}{ }^{1} \mathrm{H}$ NMR (400 MHz, $\left.\mathrm{CDCl}_{3}\right) \delta 8.23-8.19(\mathrm{~m}, 2 \mathrm{H}), 7.63(\mathrm{t}, J=7.2 \mathrm{~Hz}, 1 \mathrm{H}), 7.53-7.41(\mathrm{~m}, 4 \mathrm{H}), 7.29-7.20(\mathrm{~m}$, $3 \mathrm{H}) ;{ }^{13} \mathrm{C} \mathrm{NMR}\left(100 \mathrm{MHz}, \mathrm{CDCl}_{3}\right) \delta 165.4$ (1C), 151.2 (1C), 133.8 (1C), 130.4 (2C), 129.8 (1C), 129.7 (2C), 128.8 (2C), 126.1 (1C), 121.9 (2C); IR ( $\left.\mathrm{CDCl}_{3}\right) 3068,1730 \mathrm{~cm}^{-1}$. Phenyl 4-nitrobenzoate (entry 12, Table 1). Using procedure B, 4-nitrobenzoic acid (0.25 mmol, $42 \mathrm{mg})$, silylaryl triflate $1 \mathrm{a}(0.5 \mathrm{mmol}, 149 \mathrm{mg})$ and CsF (1.0 mmol, 152 $\mathrm{mg}$ ) afforded the indicated compound (40 mg) in a $66 \%$ yield as a white solid: $\mathrm{mp} 127$ $128{ }^{\circ} \mathrm{C}$ (lit. $\left.{ }^{13} \mathrm{mp} 128-129{ }^{\circ} \mathrm{C}\right) ;{ }^{1} \mathrm{H}$ NMR $\left(300 \mathrm{MHz}, \mathrm{CDCl}_{3}\right) \delta 8.40-8.33$ (m, 4H), 7.48$7.43(\mathrm{~m}, 2 \mathrm{H}), 7.34-7.22(\mathrm{~m}, 3 \mathrm{H}) ;{ }^{13} \mathrm{C} \mathrm{NMR}\left(75 \mathrm{MHz}, \mathrm{CDCl}_{3}\right) \delta 163.5(1 \mathrm{C}), 151.1(1 \mathrm{C})$, 150.7 (1C), 135.2 (1C), 131.5 (2C), 129.9 (2C), 126.6 (1C), 123.9 (2C), 121.6 (2C); IR $\left(\mathrm{CDCl}_{3}\right) 3111,1740 \mathrm{~cm}^{-1}$; HRMS m/z 243.0536 (calcd $\mathrm{C}_{13} \mathrm{H}_{9} \mathrm{NO}_{4}, 243.05316$ ).

Phenyl 4-methoxybenzoate (entry 13, Table 1). Using procedure B, 4-methoxybenzoic acid $(0.25 \mathrm{mmol}, 38 \mathrm{mg})$, silylaryl triflate $1 \mathbf{a}(0.5 \mathrm{mmol}, 149 \mathrm{mg})$ and CsF (1.0 mmol, 
$152 \mathrm{mg}$ ) afforded the indicated compound (55 mg) in a $96 \%$ yield as a white solid: $\mathrm{mp}$ 67-69 ${ }^{\circ} \mathrm{C}$ (lit. $\left.{ }^{14} \mathrm{mp} 67-69{ }^{\circ} \mathrm{C}\right)$. The ${ }^{1} \mathrm{H}$ and ${ }^{13} \mathrm{C}$ NMR spectra match the literature data: ${ }^{15}$ ${ }^{1} \mathrm{H}$ NMR (400 MHz, $\left.\mathrm{CDCl}_{3}\right) \delta 8.17-8.13(\mathrm{~m}, 2 \mathrm{H}), 7.43-7.39$ (m, 2H), 7.27-7.18 (m, 3H), 6.99-6.95 (m, 2H); ${ }^{13} \mathrm{C}$ NMR (100 MHz, $\left.\mathrm{CDCl}_{3}\right) \delta 165.1$ (1C), 164.1 (1C), $151.3(1 \mathrm{C})$, 132.5 (2C), 129.6 (2C), 125.9 (1C), 122.1 (1C), 122.0 (2C), 114.0 (2C), 55.7 (1C); IR

$\left(\mathrm{CDCl}_{3}\right)$ 3058, 2967, 2839, 1727, $1278 \mathrm{~cm}^{-1}$; HRMS m/z $228.0789\left(\right.$ calcd $\mathrm{C}_{14} \mathrm{H}_{12} \mathrm{O}_{3}$, 228.0786).

3-Methoxyphenyl 4-methoxybenzoate (entry 14, Table 1). Using procedure B, 4methoxybenzoic acid $(0.25 \mathrm{mmol}, 38 \mathrm{mg})$, silylaryl triflate $1 \mathbf{b}(0.5 \mathrm{mmol}, 164 \mathrm{mg})$ and $\mathrm{CsF}(1.0 \mathrm{mmol}, 152 \mathrm{mg})$ afforded the indicated compound $(63 \mathrm{mg})$ in a $98 \%$ yield as a white solid: mp 102-103 ${ }^{\circ} \mathrm{C} ;{ }^{1} \mathrm{H}$ NMR $\left(300 \mathrm{MHz}, \mathrm{CDCl}_{3}\right) \delta 8.16-8.12(\mathrm{~m}, 2 \mathrm{H}), 7.30(\mathrm{t}, J$ $=8.1 \mathrm{~Hz}, 1 \mathrm{H}), 6.98-6.95(\mathrm{~m}, 2 \mathrm{H}), 6.82-6.75(\mathrm{~m}, 3 \mathrm{H}), 3.88(\mathrm{~s}, 3 \mathrm{H}), 3.80(\mathrm{~s}, 3 \mathrm{H}) ;{ }^{13} \mathrm{C}$ NMR (75 MHz, $\left.\mathrm{CDCl}_{3}\right) \delta 165.0$ (1C), 164.1 (1C), 160.7 (1C), 152.3 (1C), 132.5 (2C), 130.0 (1C), 122.0 (1C), 114.2 (1C), 114.0 (2C), 111.9 (1C), 107.9 (1C), 55.7 (1C), 55.6 (1C); IR $\left(\mathrm{CDCl}_{3}\right)$ 3006, 2937, 2838, 1726, 1605, $1255 \mathrm{~cm}^{-1}$; HRMS m/z 258.0895 (calcd $\left.\mathrm{C}_{15} \mathrm{H}_{14} \mathrm{O}_{4}, 258.0892\right)$.

3-Methoxyphenyl 4-bromobenzoate (entry 15, Table 1). Using procedure B, 4bromobenzoic acid $(0.25 \mathrm{mmol}, 50 \mathrm{mg})$, silylaryl triflate $\mathbf{1 b}(0.5 \mathrm{mmol}, 164 \mathrm{mg})$ and CsF $(1.0 \mathrm{mmol}, 152 \mathrm{mg})$ afforded the indicated compound $(68 \mathrm{mg})$ in an $89 \%$ yield as a white solid: mp 59-61 ${ }^{\circ} \mathrm{C} ;{ }^{1} \mathrm{H}$ NMR $\left(300 \mathrm{MHz}, \mathrm{CDCl}_{3}\right) \delta 8.07-8.03(\mathrm{~m}, 2 \mathrm{H})$, 7.67-7.63 $(\mathrm{m}, 2 \mathrm{H}), 7.32(\mathrm{t}, J=8.1 \mathrm{~Hz}, 1 \mathrm{H}), 6.85-6.76(\mathrm{~m}, 3 \mathrm{H}), 3.23(\mathrm{~s}, 3 \mathrm{H}) ;{ }^{13} \mathrm{C} \mathrm{NMR}(75 \mathrm{MHz}$, $\left.\mathrm{CDCl}_{3}\right) \delta 164.6(1 \mathrm{C}), 160.8(1 \mathrm{C}), 151.9$ (1C), 132.2 (2C), 131.8 (2C), 130.1 (1C), 129.0 (1C), 128.7 (1C), 114.0 (1C), 112.2 (1C), 107.8 (1C), 55.7 (1C); IR ( $\left.\mathrm{CDCl}_{3}\right)$ 2933, 2834, 1729, 1585, $1254 \mathrm{~cm}^{-1}$; HRMS m/z 305.9897 (calcd $\mathrm{C}_{14} \mathrm{H}_{11} \mathrm{BrO}_{3}, 305.9891$ ).

Phenyl $o$-iodobenzoate (entry 16, Table 1). Using procedure B, 2-iodobenzoic acid $(0.25 \mathrm{mmol}, 62 \mathrm{mg})$, silylaryl triflate $1 \mathrm{a}(0.5 \mathrm{mmol}, 149 \mathrm{mg})$ and CsF $(1.0 \mathrm{mmol}, 152$ $\mathrm{mg}$ ) afforded the indicated compound $(63 \mathrm{mg})$ in a $78 \%$ yield as a colorless oil. The ${ }^{1} \mathrm{H}$ and ${ }^{13} \mathrm{C}$ NMR spectra match the literature data: ${ }^{16}{ }^{1} \mathrm{H}$ NMR $\left(300 \mathrm{MHz}, \mathrm{CDCl}_{3}\right) \delta 8.08$ $8.02(\mathrm{~m}, 2 \mathrm{H}), 7.51-7.41(\mathrm{~m}, 3 \mathrm{H}), 7.31-7.19(\mathrm{~m}, 4 \mathrm{H}) ;{ }^{13} \mathrm{C} \mathrm{NMR}\left(75 \mathrm{MHz}, \mathrm{CDCl}_{3}\right) \delta 165.1$ (1C), 150.9 (1C), 141.9 (1C), 134.4 (1C), 133.4 (1C), 131.7 (1C), 129.7 (2C), 128.3 (1C), 
126.3 (1C), 121.8 (2C), 94.8 (1C); IR $\left(\mathrm{CDCl}_{3}\right) 3063,1743 \mathrm{~cm}^{-1}$; HRMS m/z 323.9653 (calcd $\mathrm{C}_{13} \mathrm{H}_{9} \mathrm{IO}_{2}, 323.9647$ ).

\section{References}

(1) Pena, D.; Perez, D.; Guitian, E.; Castedo, L. J. Am. Chem. Soc. 1999, 121, 5827.

(2) Yoshida, H.; Sugiura, S.; Kunai, A. Org. Lett. 2002, 4, 2767.

(3) Ma, D.; Cai, Q. Org. Lett. 2003, 5, 3799.

(4) (a) Haga, N.; Takayanagi, H. J. Org. Chem. 1996, 61, 735. (b) Nyquist, R. A.;

Hasha, D. L. Appl. Spectrosc. 1991, 45, 1649.

(5) Marcoux, J.-F.; Doye, S.; Buchwald, S. L. J. Am. Chem. Soc. 1997, 119, 10539.

(6) (a) Nyquist, R. A.; Hasha, D. L. Appl. Spectrosc. 1991, 45, 849. (b) Yeager, G. W.; Schissel, D. N. Synthesis 1991, 1, 63.

(7) Paradisi, C. J. Org. Chem. 1983, 48, 3022.

(8) Pouchert, C. J.; Campbell, J. R. "The Aldrich Library of NMR Spectra"; Aldrich Chemical Co.: Milwaukee, WI, 1974; Vol. 5, p 186B.

(9) Abramovitch, R. A.; Alvernhe, G.; Bartnik, R.; Dassanayake, N. L.; Inbasekaran, M. N.; Kato, S. J. Am. Chem. Soc. 1981, 103, 4558.

(10) Brewster, R. Q.; Strain, F. J. Am. Chem. Soc. 1934, 56, 117.

(11) Glatzhofer, D. T.; Roy, R. R.; Cossy, K. N. Org. Lett. 2002, 4, 2349.

(12) Lee, C. K.; Yu, J. S.; Kim, S. H. J. Heterocycl. Chem. 1998, 35, 835.

(13) Tarbell, D. S.; Price, J. A. J. Org. Chem. 1957, 22, 245.

(14) Hsshimoto, S. Furukawa, I. Bull. Chem. Soc. Jpn. 1981, 54, 2227.

(15) Bauerova, I.; Ludwig, M. Collect. Czech. Chem. Commun. 2000, 65, 1777.

(16) Larock, R. C.; Doty, M. J.; Han, X. J. Org. Chem. 1999, 64, 8770. 


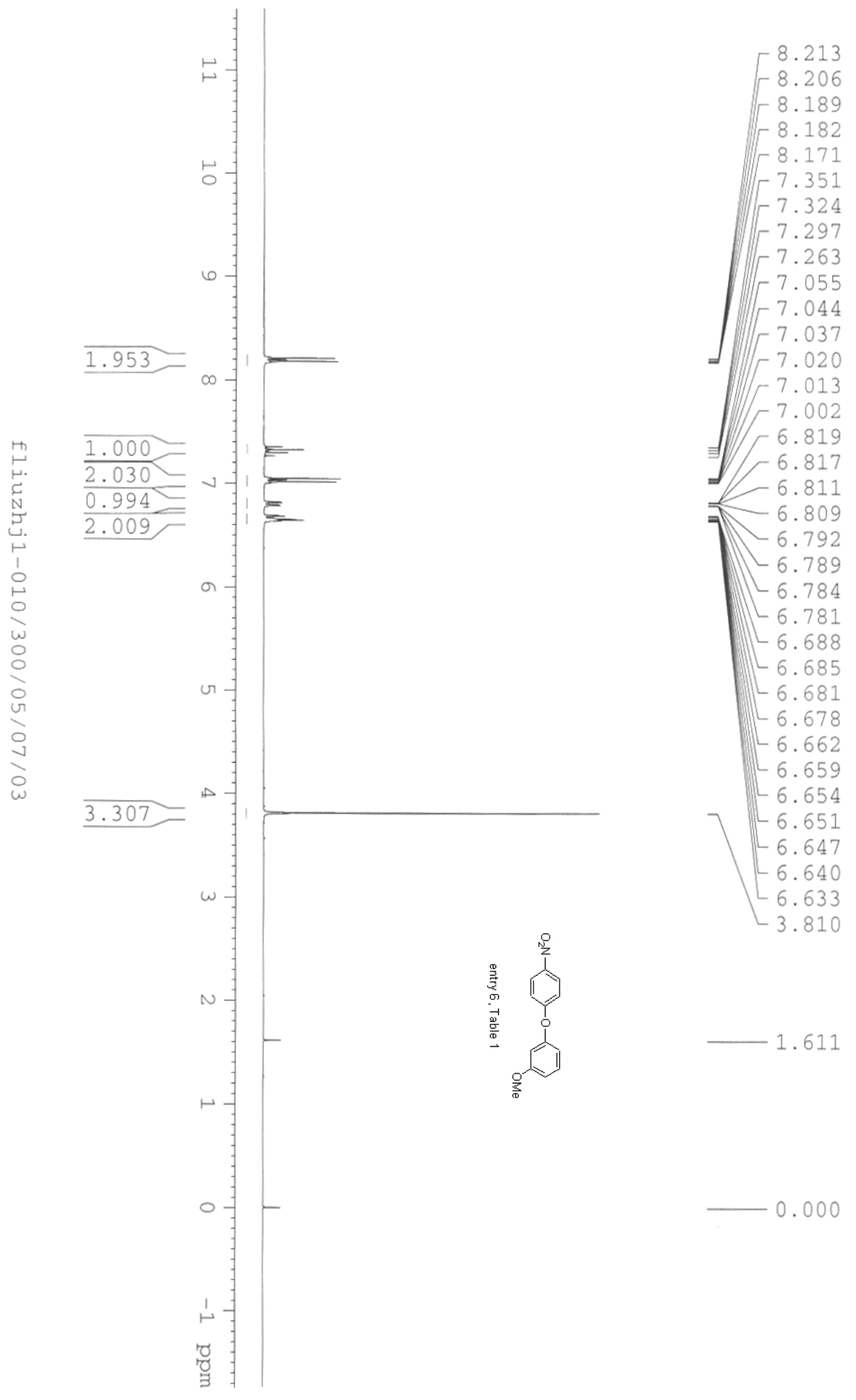



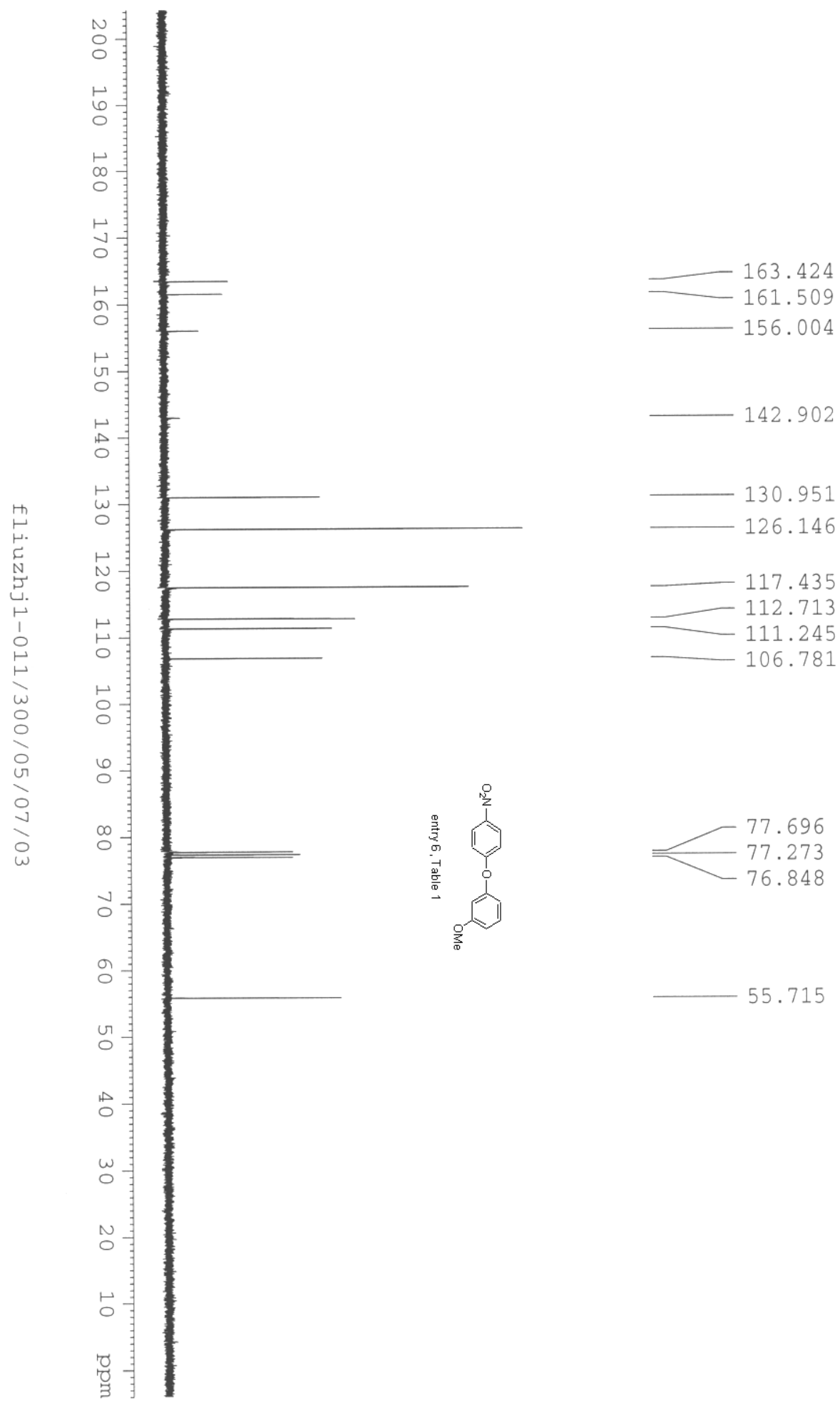

55.715 


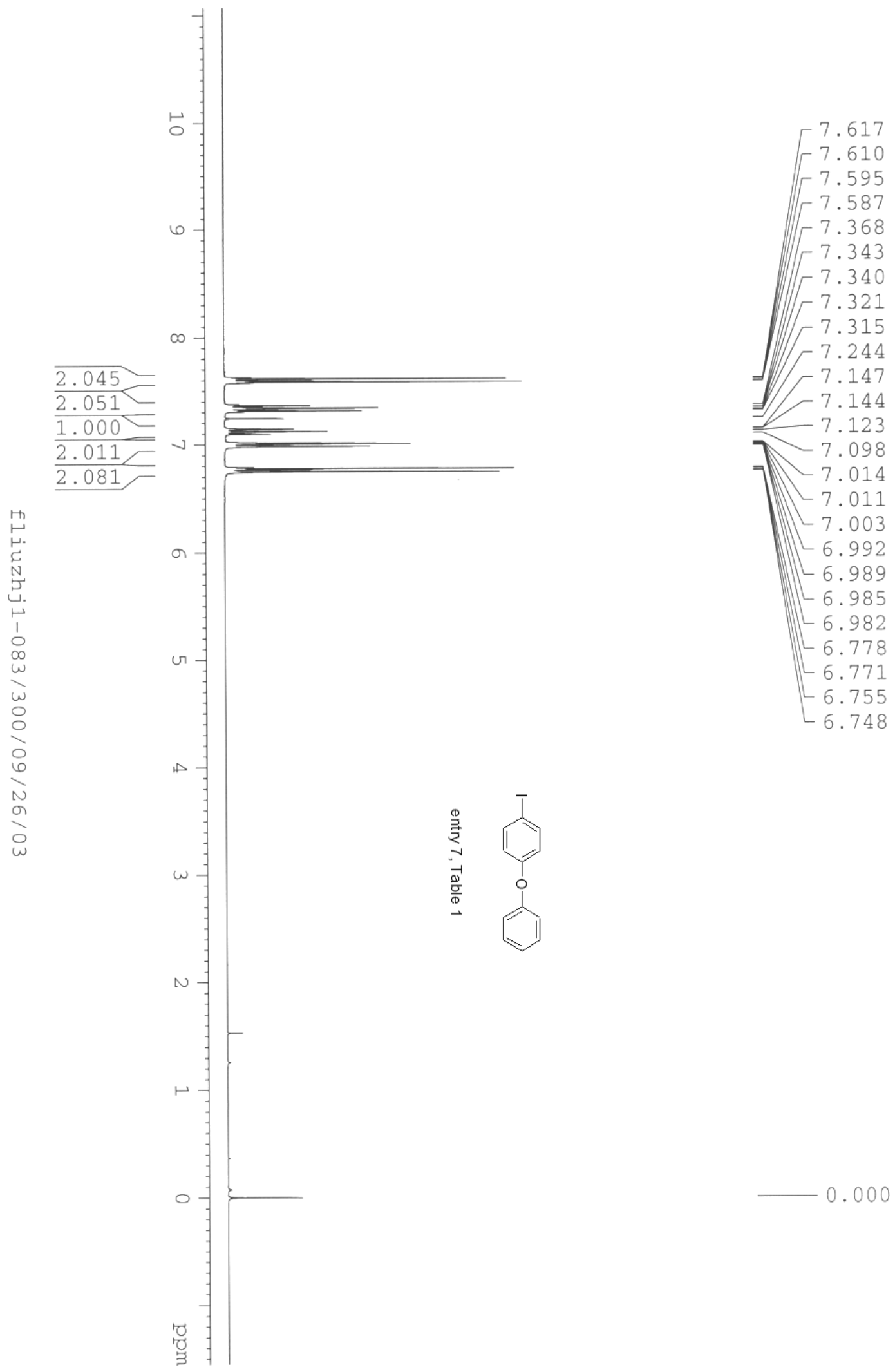




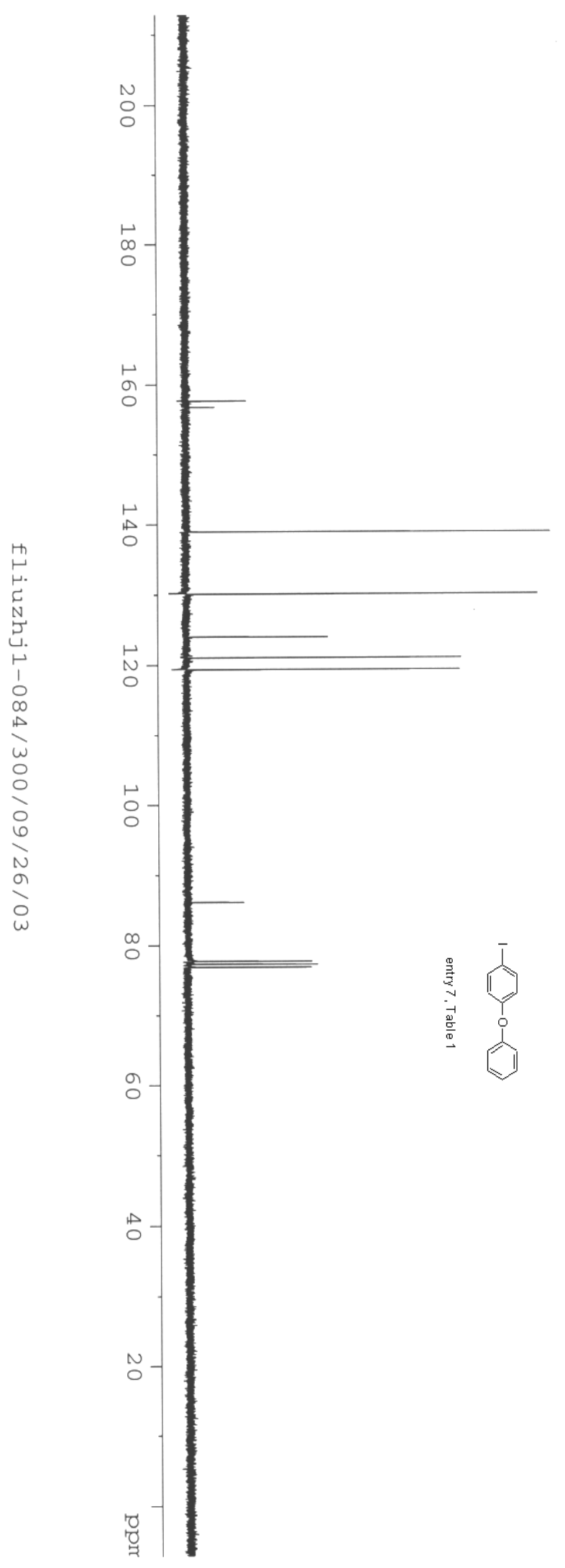

157.690 156.778

138.885

130.131

124.032 121.072 119.390

$-86.120$

77.696

77.272

76.849

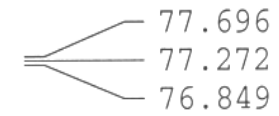




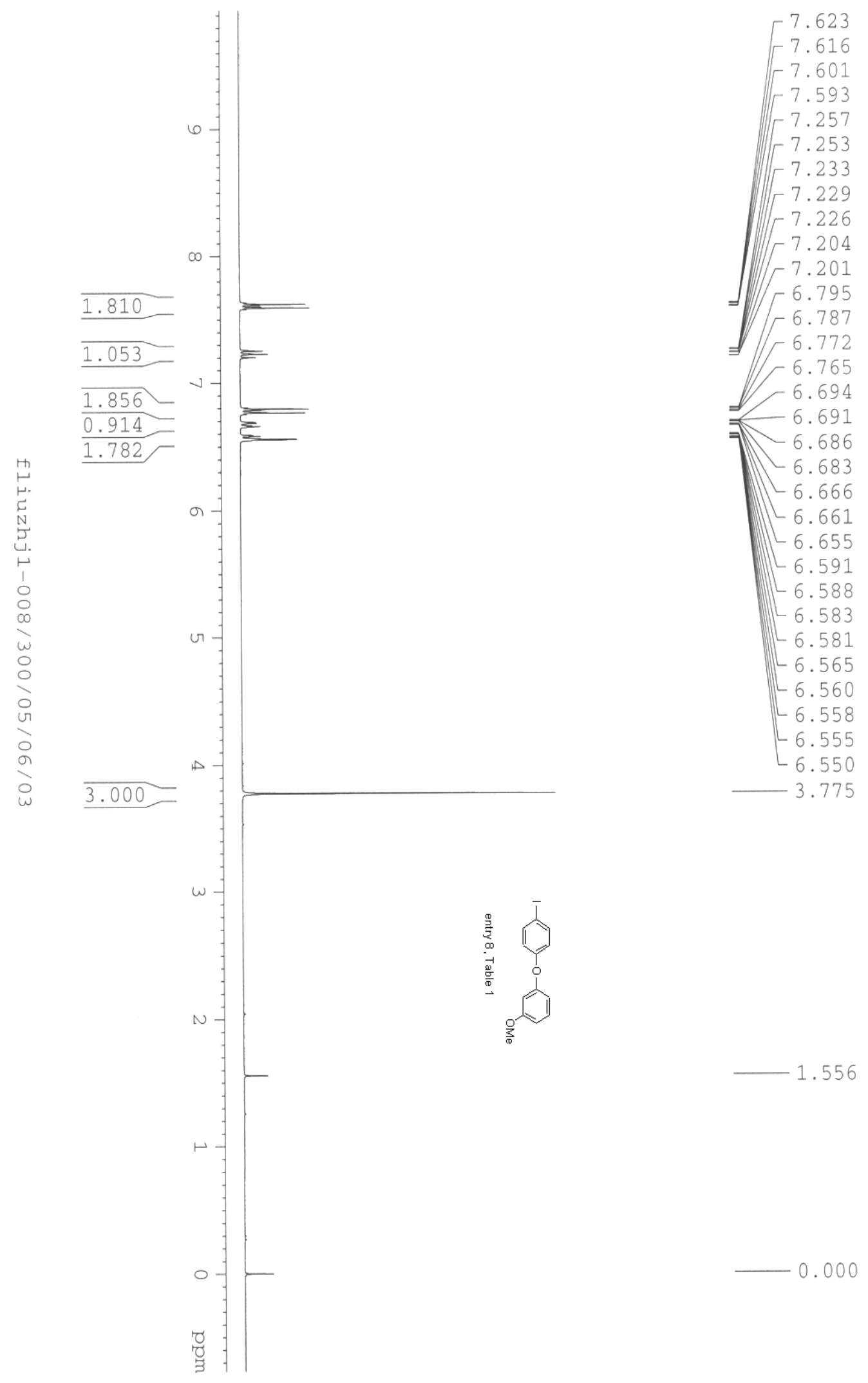




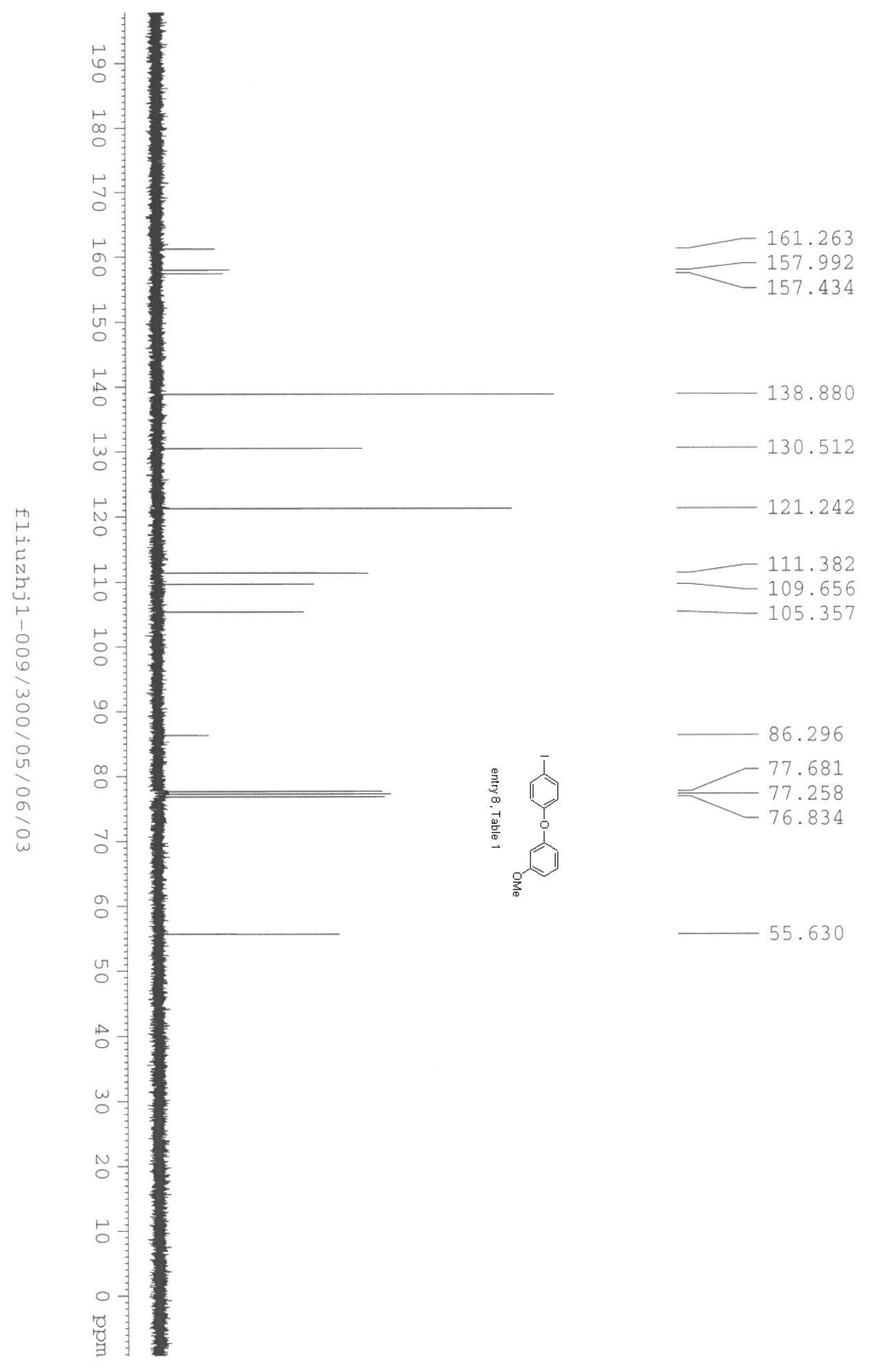



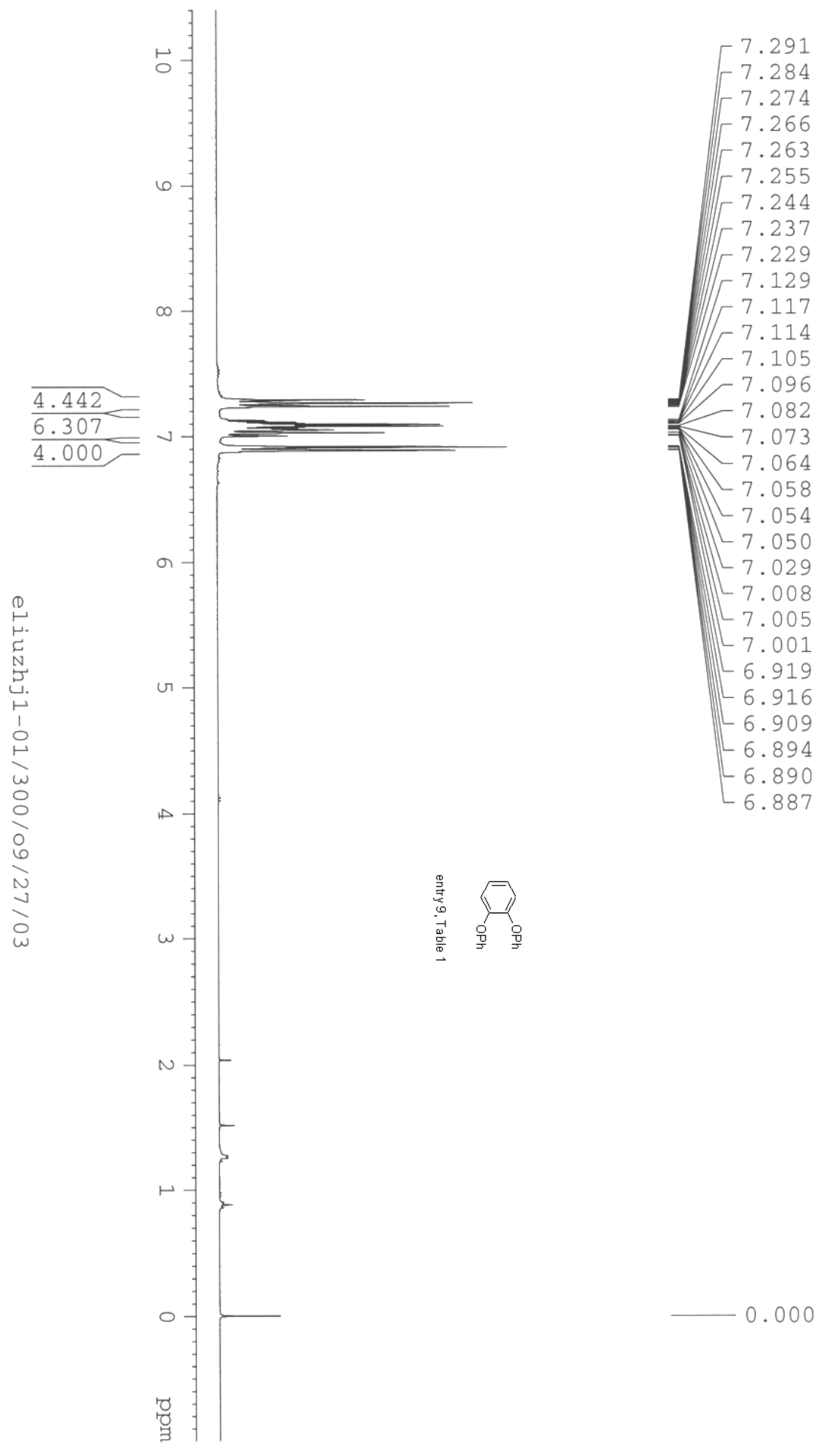

$-0.000$ 


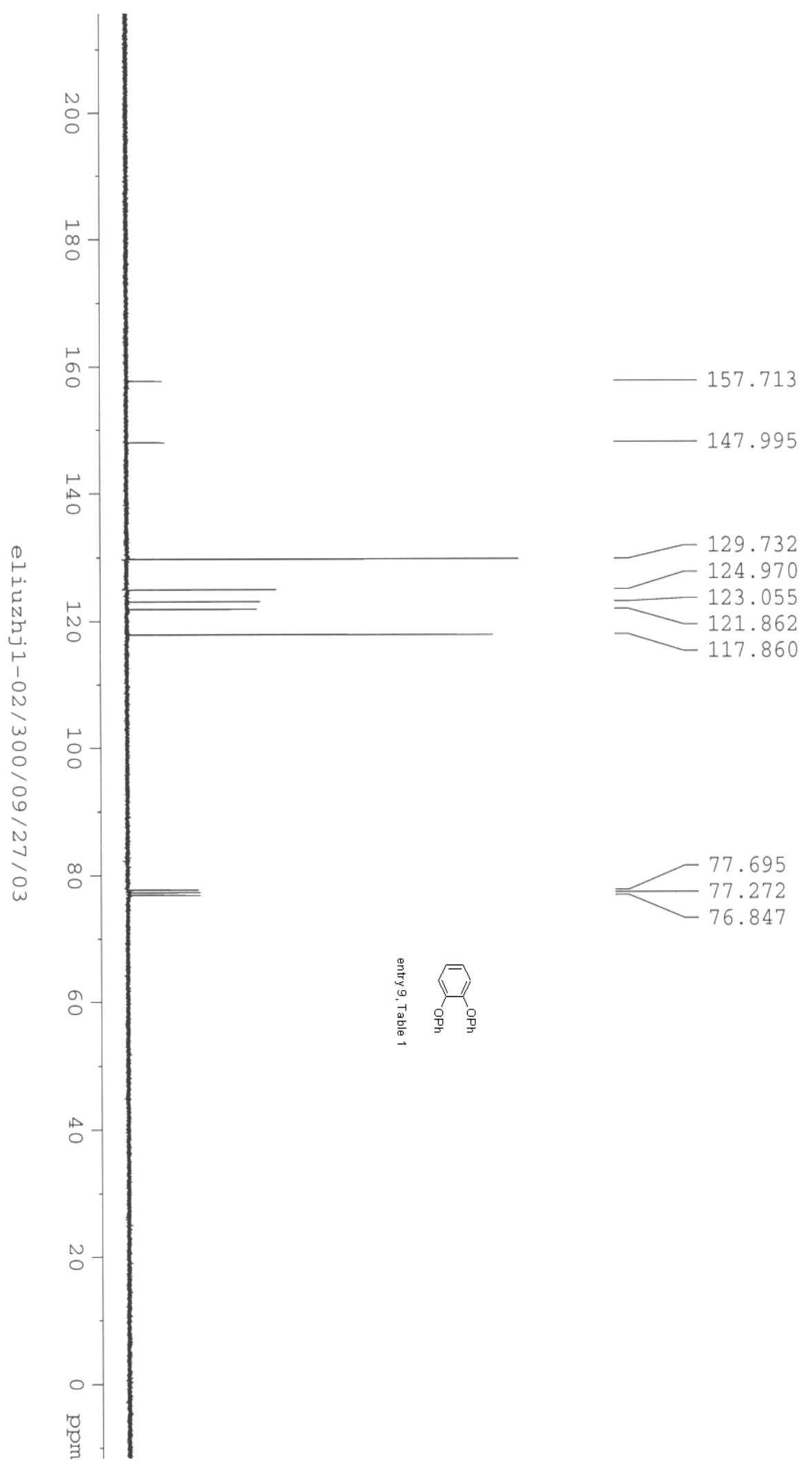




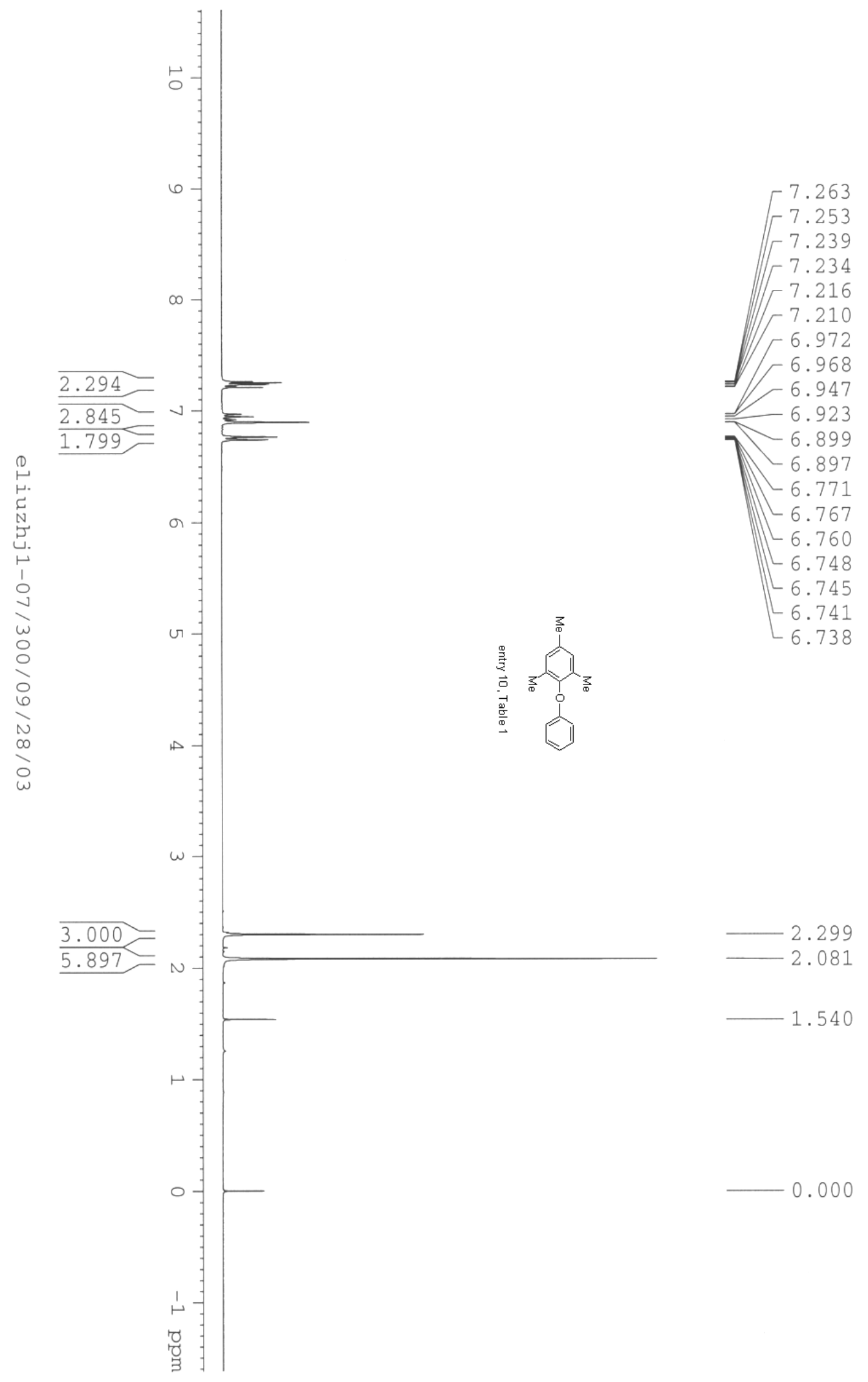




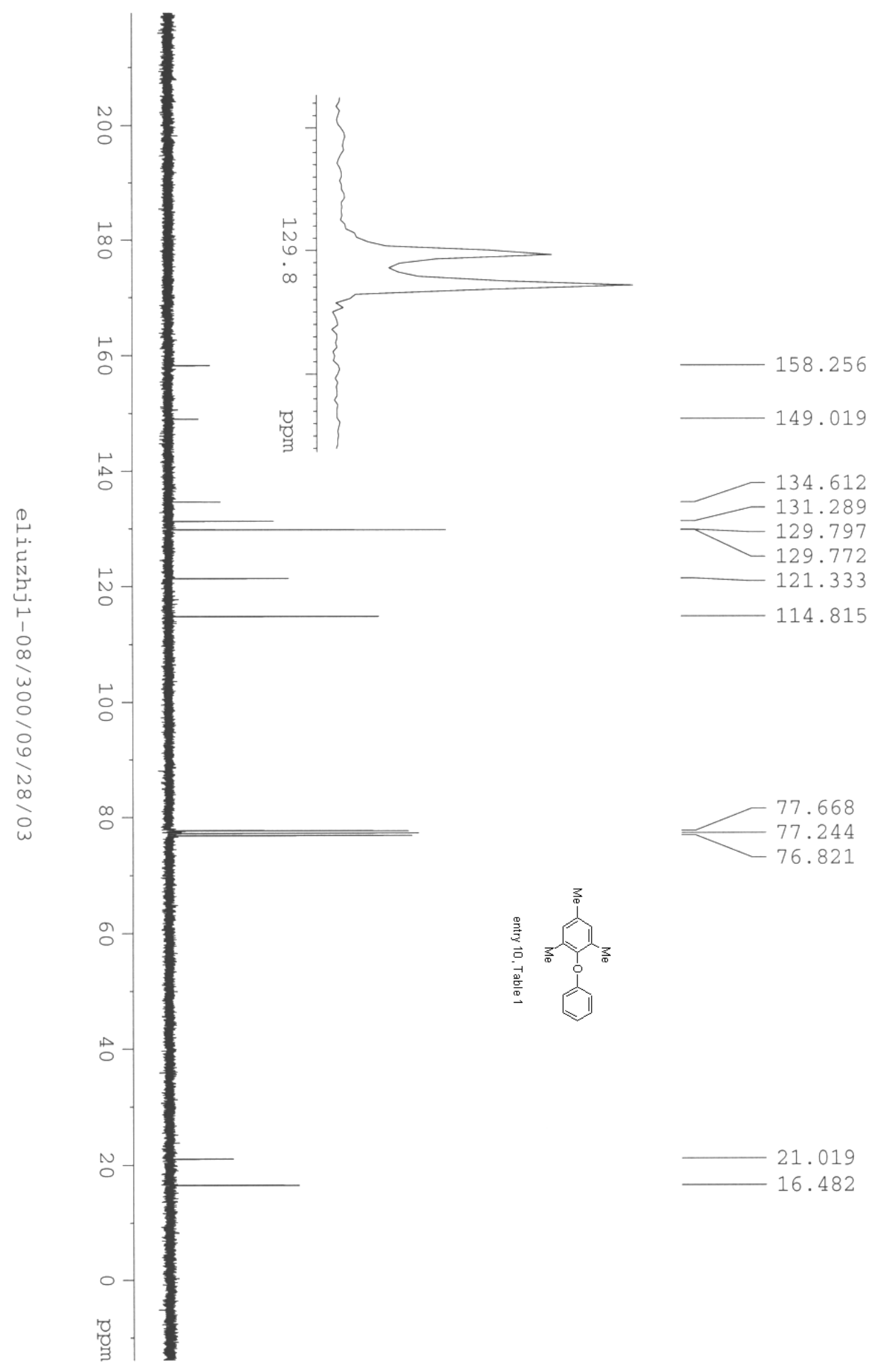




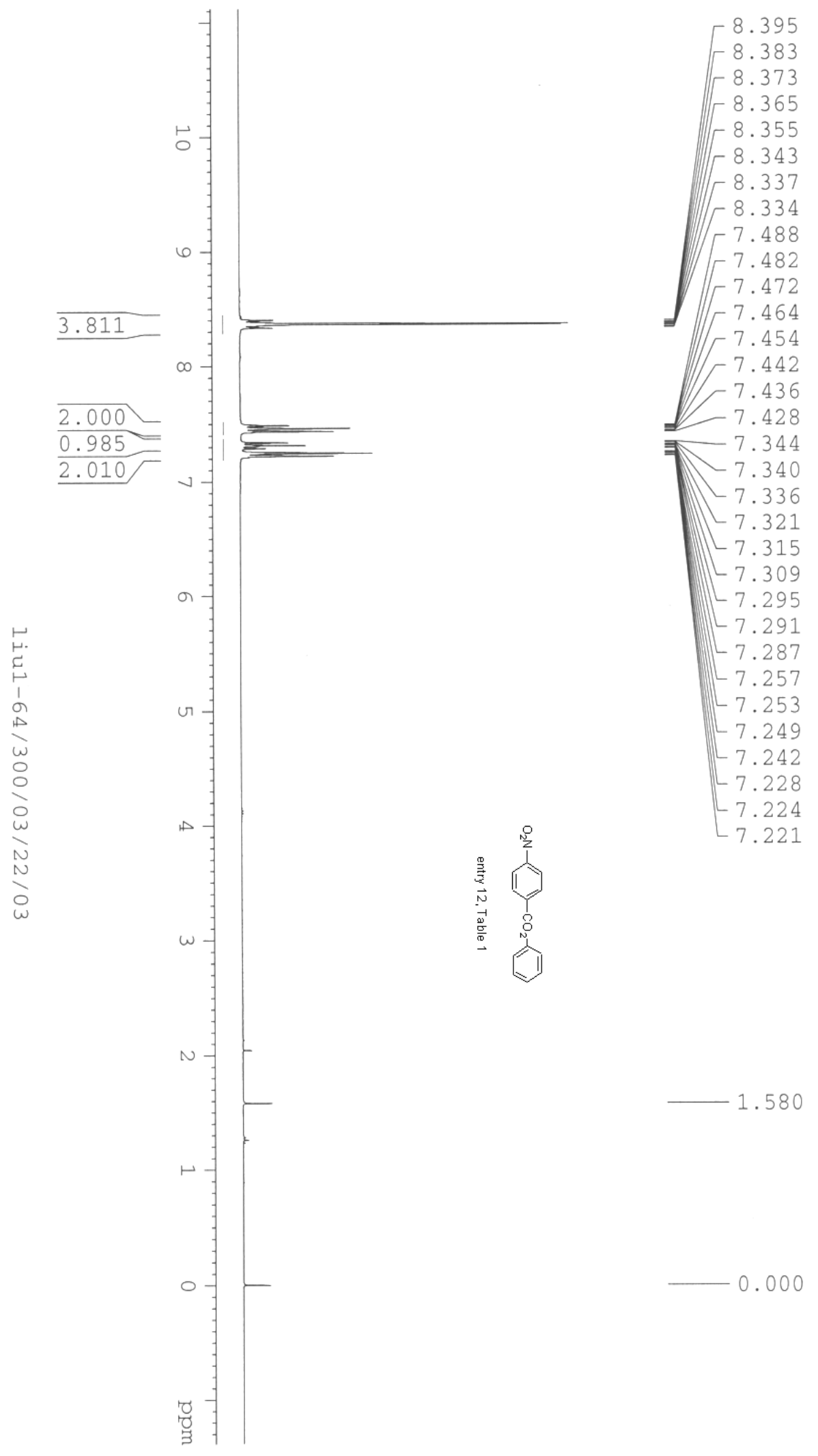



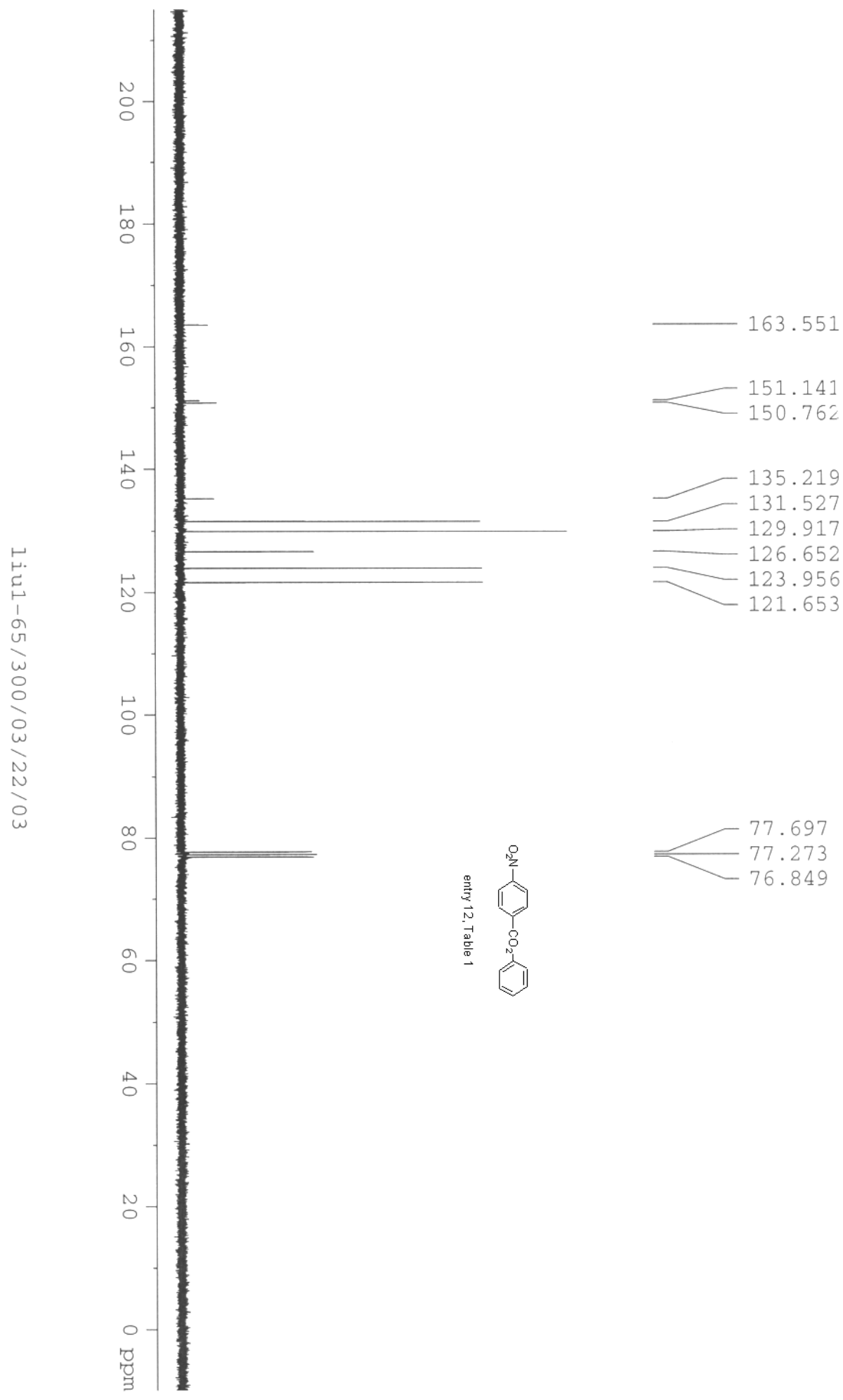

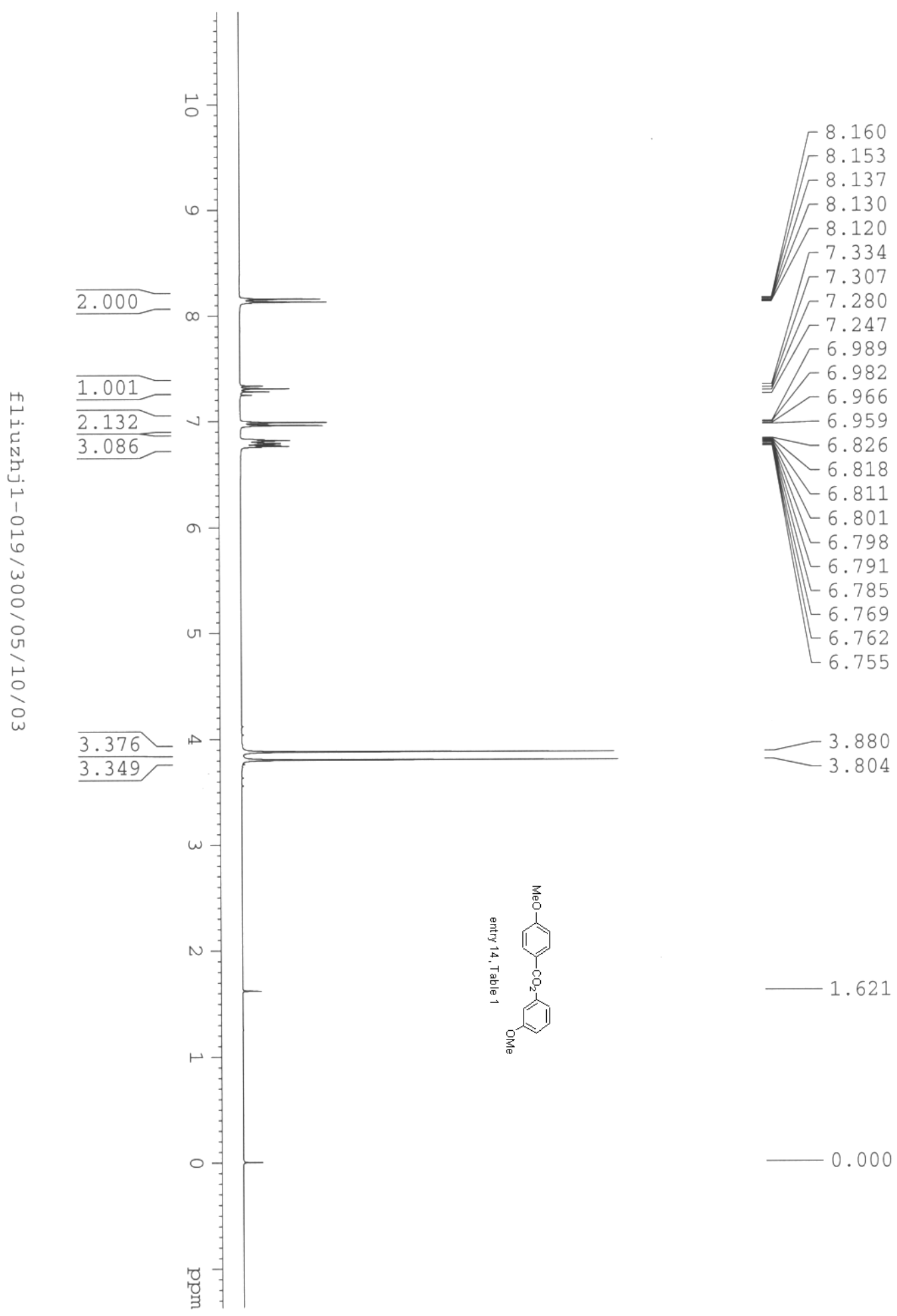

0.000 

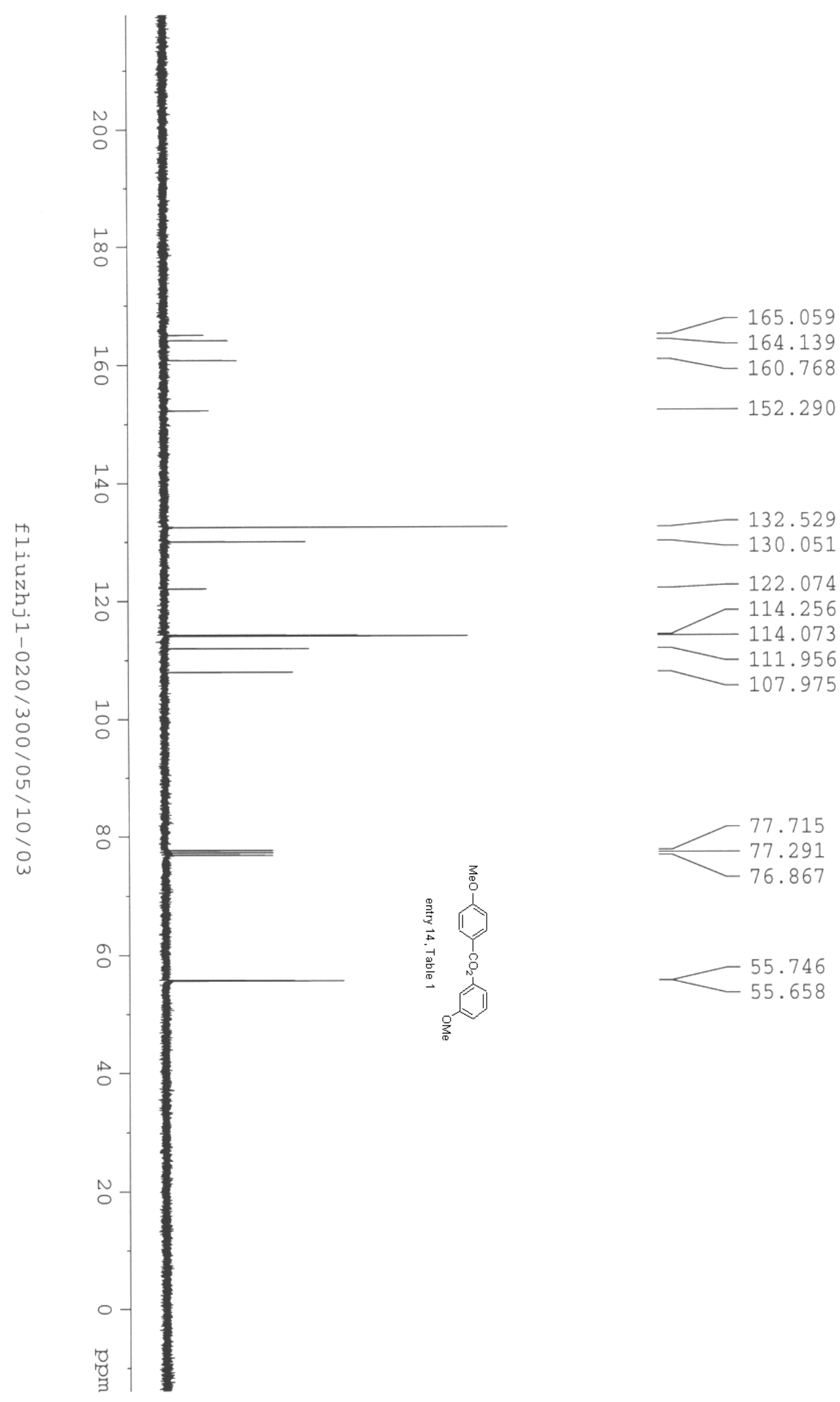


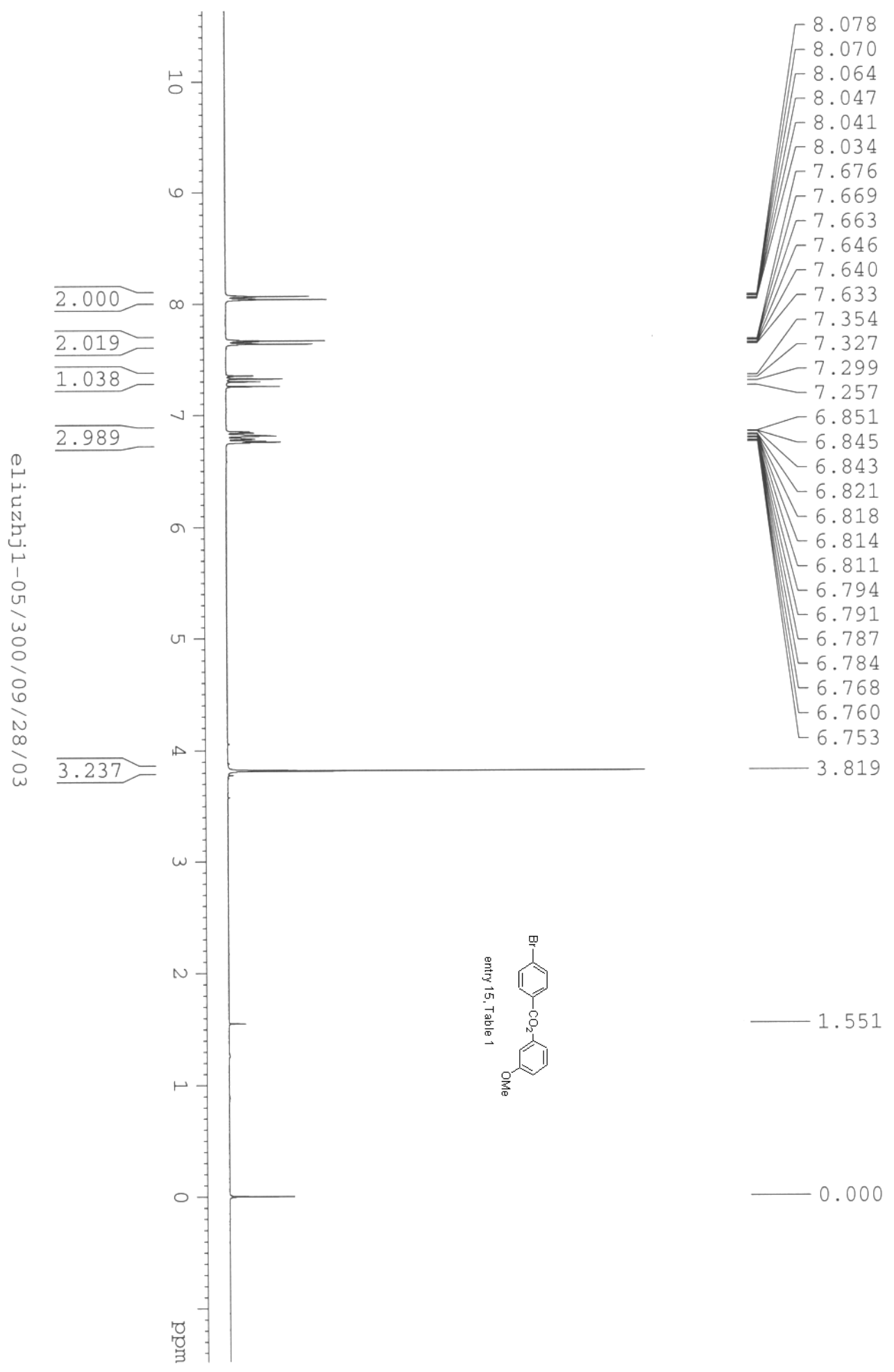




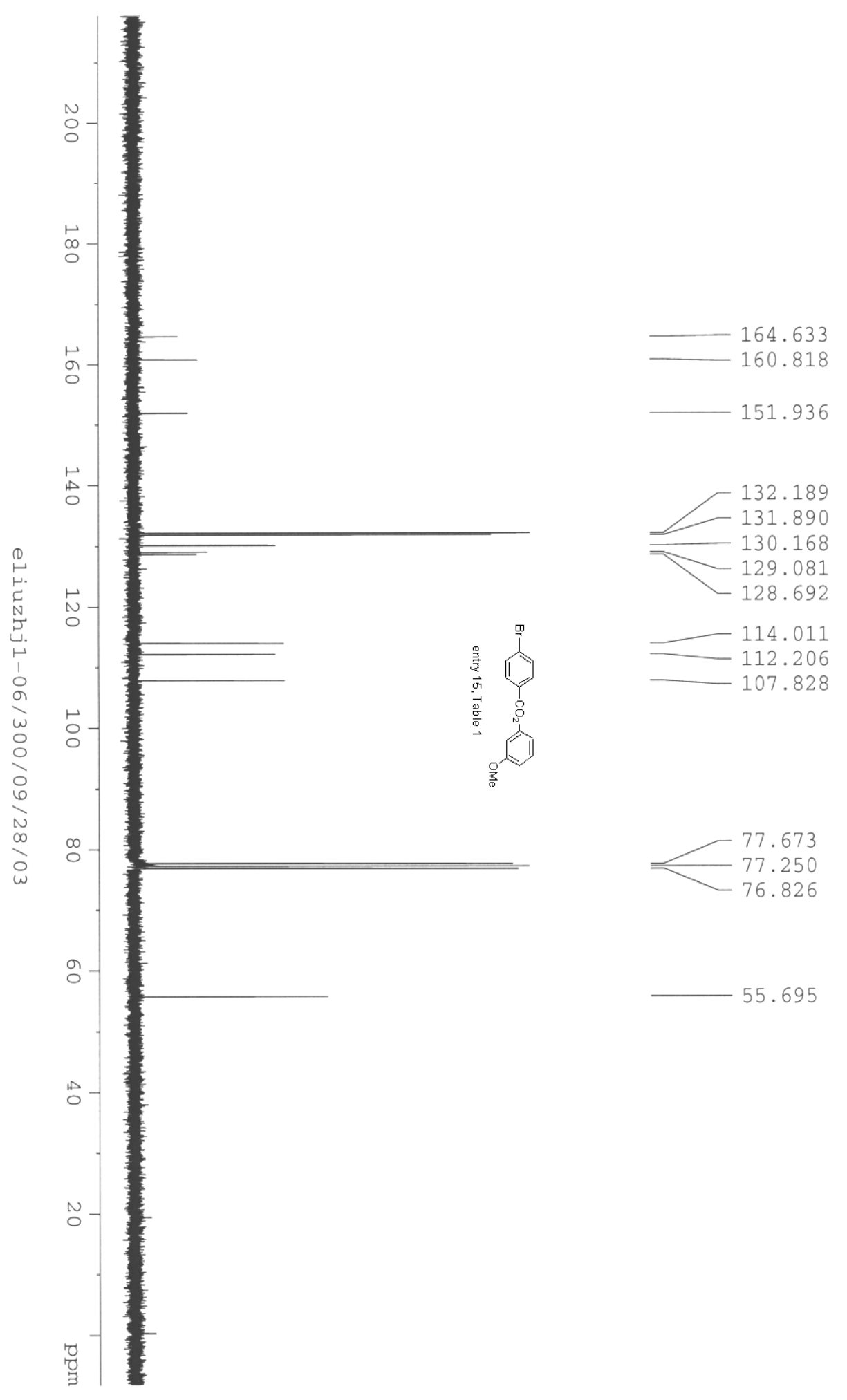

